\title{
A LP-RR Principle-Based Admission Control for a Mobile Network
}

\author{
B. P. Vijay Kumar, Student Member, IEEE, and Pallapa Venkataram, Senior Member, IEEE
}

\begin{abstract}
In mobile networks, the traffic fluctuation is unpredictable due to mobility and varying resource requirement of multimedia applications. Hence, it is essential to maintain traffic within the network capacity to provide service guarantees to running applications. This paper proposes an admission control (AC) scheme in a mobile cellular environment supporting hand-off and new application traffic. In the case of multimedia applications, each applications has its own distinct range of acceptable quality of service $(\mathrm{QoS})$ requirements (e.g., packet loss, delay, jitter, etc.). The network provides the service by maintaining the application specified QoS range. We propose a linear programming resource reduction (LP-RR) principle for admission control by maintaining QoS guarantees to existing applications and to increase the percentage of admission to hand-off and new applications. Artificial neural networks (ANNs) are used to solve linear programming problem, which facilitates in real time admission control decision in the practical systems.

We present an analytical model and results for the proposed admission control (AC) scheme with resource reduction principle and a simulation study of the admission control for performance evaluation.

The simulation results demonstrate that the proposed AC scheme performs well in terms of increasing the number of admitted applications and maintains higher percentage of resource utilization. The suggested principle also shown that it is appropriate for the fair resource allocation with improved resource utilization.
\end{abstract}

Index Terms-Linear programming, neural network, quality of service $(\mathrm{QoS})$, resource reduction.

\section{INTRODUCTION}

$\mathbf{F}$ UTURE mobile networks will be required to carry the traffic generated by wide range of services. These services will have very diverse traffic flow characteristics and performance requirement. Some of the many factors that have led to this diversified nature of high-speed network traffic are services integration, broad range of compression schemes and broad range of applications. A key characteristics of multimedia type application services is that they require different quality of service (QoS) guarantees.

The population of the mobile users is growing at a rapid rate and that the applications are becoming more bandwidth intensive; hence, the traffic control of mobile networks has become a necessary element of mobile communications. Due to the limitations of the radio spectrum, the wireless systems use micro/picocellular architectures in order to provide a higher capacity [1].

Manuscript received January 19, 2001; revised August 8, 2002.

The authors are with the PET unit, Department of Electrical Communication Engineering, Indian Institute of Science, Bangalore, India (e-mail vijaybp@ece.iisc.ernet.in; pallapa@ece.iisc.ernet.in).

Digital Object Identifier 10.1109/TSMCC.2002.806071
Because of the small coverage area of micro/pico-cells and the characteristics of the multipath and shadow fading radio environment, hand-off events in the systems will occur at a much higher rate as compared to macro-cellular systems. Frequent hand-offs in wireless/mobile networks and broad range of service requirement of multimedia applications introduce a new paradigm in the area of admission control and congestion. Also, in such scenario, the mobile networks have to support multiple classes of services with widely different traffic characteristics and QoS requirements.

To ensure the promised QoS can be met, applications declare their traffic characteristics in terms of traffic descriptor parameters along with the QoS expectations, which can be mapped to appropriate traffic classes supported by the base station switch. With the information provided by the applications at the connection setup time, the network uses an admission control (AC) to determine whether the new request for a connection should be accepted or rejected. The decision made by the AC to accept a new application is based on the availability of resources (bandwidth, buffers, etc.,) to ensure the resources (QoS) requested by the new application can be guaranteed, without degrading the QoS requirement of applications that have already been scheduled.

In this paper, an admission control scheme is proposed to provide service guarantees for multimedia traffic carried in micro/ pico-cellular mobile networks. We also present an analytical results and proofs for the proposed AC scheme.

The proposed scheme is restricted to the applications running within a cell without loss of generality, by considering the local information of unused resources and allocated resources to the running applications to determine whether to accept or reject the requesting applications for scheduling based on their QoS requirement. The scheme uses a linear programming resource reduction (LP-RR) principle for AC to minimize average rejection of hand-off and new applications and to maximize the resource utilization. The linear programming (LP) problem is solved by using artificial neural networks (ANNs), which are inherently parallel and distributed and this aids in real time information processing and decision making capability.

The rest of the paper is organized as follows. Section II surveys the related work. Admission control and QoS specifications for the traffic services are described in Section III. Some of the definitions that are followed in this paper are illustrated in Section IV, along with LP-RR principle and proposed LP-RR based AC scheme. The analytical model with results are described in Section V. The simulation model along with the simulation results are given in Section VI. Finally, Section VII provides the concluding remarks. 


\section{RELATED WORK}

Most of the existing admission control schemes to guarantee QoS consider the following methodology to prevent overloading of mobile networks. In [2], AC for integrated voice and data traffic in packet radio networks was investigated. The admission control policy is based on a predetermined threshold value of either the mean delay or the packet loss probability for data traffic. The admission control scheme, proposed in [3], employs different resource sharing schemes to allocate resources to different classes of traffic. A distributed admission control scheme given in [4] is based on both the number of existing connections in a cell where a connection request is generated and the number of connections in the adjacent cells. Admission control is performed at each base station in a distributed manner and it considers only a single traffic type, i.e., voice traffic. An AC based on dynamic channel assignment was proposed in [5]. In this scheme, network traffic conditions are first evaluated in the AC phase and then channels are assigned accordingly to new calls.

The works in [6]-[8], consider bandwidth reservation for hand-off applications to guarantee high connectivity of admitted calls. A fixed number of channels in each cell is reserved exclusively for hand-offs. If none of the reserved channels are available, the hand-off applications are queued up with a minimum queuing delays with some threshold value. If the queuing delay exceeds the threshold value the hand-off application is as good as dropped. In [9], it is proposed and analyzed a class of AC algorithms based on reward optimization for multimedia servers designed to provide on-demand services to clients in an environment where workload characteristics of clients can change dynamically. These AC algorithms are developed based on two choices, namely "deterministic" or "best-effort" regarding QoS, and "priority reservation" or "no-priority reservation" regarding reservation control.

Levine et al. [10] have proposed predictive resource allocation and $\mathrm{AC}$ schemes based on a concept, called shadow cluster, to improve the resource utilization and reduction in call dropping probability. The shadow cluster concept is used in AC to allow predictive resource allocation. In this scheme, mobile terminals inform the base stations in neighboring cells of their bandwidth requirements, position and movement parameters at call setup time. Based on this information, base stations predict future demands, reserves resources accordingly, and admit only those mobile terminals that can be supported adequately. An idea of using user mobility patterns is employed in this scheme and its effectiveness has been demonstrated in the simulation study.

A batch and priority based AC is proposed [11] to support multimedia services having diverse QoS (delay, throughput) requirement, in mobile ad hoc networks. A multiclass connection AC policy is proposed in [12] that supports cell loss and delay requirement for high speed ATM switches. A closed form formulae to calculate the bandwidth required to meet the guarantees on QoS is derived for high level of resource utilization and real-time AC. A service model for real-time services to mobile hosts that can accommodate highly adaptive applications as well as applications which requires mobility independent QoS guarantee is presented in [13], and a measurement-based AC scheme for this model is proposed that has achieved a very high utilization of network bandwidth.
The QoS-based resource allocation model (Q-RAM) for satisfying multiple quality-of-service dimensions in a resourceconstrained environment is proposed in [14]. Using this model, available system resources can be apportioned across multiple applications such that the net utility that accrues to the end-users of those applications is maximized.

Three novel AC algorithms are presented in [15]. The first algorithm uses the weighted sum of the number of calls in progress in various cells when making the admission decision. The second computes a probability index, which reflects the forced termination probability of a new call arrival and admits those calls with low probability indexes. The third, which is a hybrid, scheme combines above two approaches.

A connection AC for heterogeneous services in wireless multiple access networks are investigated in [16] that makes use of user mobility information to reserve an appropriate amount of system resources for potential hand-off connections to achieve low hand-off connection dropping rate.

In the proposed scheme, we consider a mobile network that can support heterogeneous applications with diverse resource requirements. The proposed scheme neither looks into the number of running applications in the neighboring cells nor it reserve any resources for hand-off applications to take AC decision. Instead, a fair reduction of resources (like buffer, bandwidth, etc.) allocated to running applications is carried out (without disturbing their earlier contracted service guarantee), if the requesting applications demand is more than the available resources. Also, the main objective of the AC scheme is to reduce the rate of hand-off application failures with better utilization of the resources.

\section{ADMISSION CONTROL}

Admission control in a mobile network environment prevents congestion by limiting the admission of new applications in order to provide the guaranteed QoS for the existing applications by allocating the resources. The objective of AC scheme is to maintain the sum of traffic loads in a cell (due to both new and hand-off applications) to below some predetermined congestion level. In order to achieve this, whenever a set of multimedia applications request for certain quantity of different resources and if the available quantity of resources are less than the sum of minimum requirements of all the requesting applications, then some of the applications must be dropped, and one could even allow some applications to have less than their minimum resource requirement. Then comes the problem of which applications to drop and which one to accept, but it is feasible to accept previously running applications of user moving from its neighboring cells. Since premature termination of established connections is usually more objectionable than rejection of a new application request, the mobile network must give higher priority to hand-off applications as compared to new applications. The proposed AC scheme consider the similar kind of priorities for hand-off and new applications.

\section{A. QoS Specifications for the Traffic Services}

The QoS requirements and traffic descriptors dictates that different application traffic classes be accorded different priorities 
with different resource requirement. Here, we consider that the traffic characteristics and the desired QoS can together be represented by the quantity of resource requirement of an application and their tolerance specification by maximum and minimum resource requirement bound. The QoS requirements dictates that different traffic classes be accorded different priorities, which have wide variation in maximum and minimum value of resource requirement bound.

The bounded span of resource requirement varies from one traffic class to another, for example, a real-time application traffic class requires a nominal tolerance in delay, (because of tightly constrained transfer delay and delay variation); hence, the maximum and minimum resource requirement bounds are almost close to each other, and it depends on the QoS requirement for each of the three multimedia substreams. In case of nonreal-time application traffic, the QoS parameter tolerance (i.e., delay insensitive) has made a maximum and minimum resource requirement bound to vary to a large extent, and sometimes, the application can tolerate a minimum resource requirement value of almost zero. For example, the case of the FTP application, which is a nonreal-time application, can transfer the data at peak rate or can be stalled for a while (with data rate of zero megabits per second) until free resources are available.

\section{LP-RR BASED ADMISSION CONTROL SCHEME}

\section{A. Definitions}

We introduce some of the definitions that are used in this paper.

Definition 1: Network Resources are a set of resources $\{1,2, \ldots, q\}$ available for users in a mobile cell, the set of parameters $\left\{P_{1}, P_{2}, \ldots, P_{q}\right\}$ indicate their maximum capacities, respectively.

For example, if the $j$ th resource is the bandwidth having maximum capacity of $100 \mathrm{Mb} / \mathrm{s}$, then $P_{j}=100$.

Definition 2: QoS requirements and allocated resources for an $i$ th application $A_{i}$ is defined as $(Q A)_{A_{i}}$, which uses the resources $\{1,2, \ldots q\}$.

$(Q A)_{A_{i}}=\left\{A_{i 1}, A_{i 2}, \ldots, A_{i q}\right\}$, where $A_{i j}=$ The amount of $j$ th resource required by the application $A_{i}$.

Since applications are declaring the resource requirement in the range of maximum and minimum, then the $j$ th resource requirement and the system allocates a value in between for an application $A_{i}$, which may be given as $A_{i j}=\left(A_{i j}^{\max }, A_{i j}^{\text {alloc }}, A_{i j}^{\min }\right)$ and its corresponding $(Q A)_{A_{i}}$, which uses $q$ resources, is as follows.

$$
\begin{aligned}
(Q A)_{A_{i}}=\{ & \left(A_{i 1}^{\max }, A_{i 1}^{\text {alloc }}, A_{i 1}^{\min }\right) \\
& \left.\left(A_{i 2}^{\max }, A_{i 2}^{\text {alloc }}, A_{i 2}^{\min }\right), \ldots,\left(A_{i q}^{\max }, A_{i q}^{\text {alloc }}, A_{i q}^{\min }\right)\right\}
\end{aligned}
$$

where

$A_{i j}^{\min } \quad$ minimum value of $j$ th resource requirement of $i$ th application;

$A_{i j}^{\max }$ maximum value of $j$ th resource requirement of $i$ th application;

$A_{i j}^{\text {alloc }}$ allocated value of $j$ th resource to $i$ th application.

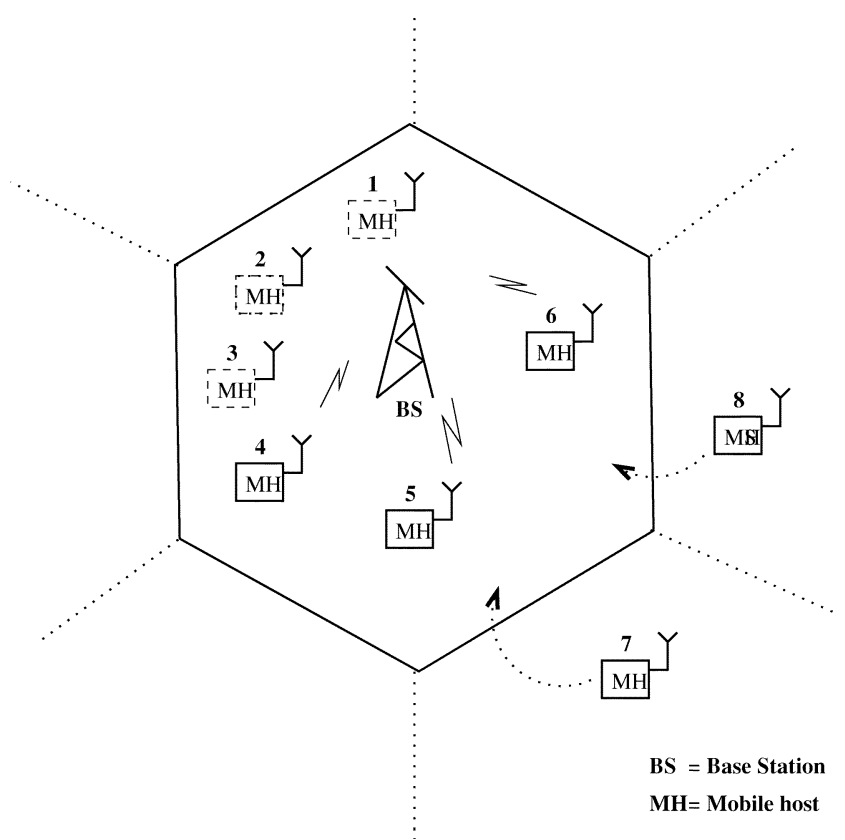

Fig. 1. Single cell network model.

For example, if an $i$ th application's requirement for bandwidth (say, $j$ th resource) of maximum value of $64 \mathrm{~Kb} / \mathrm{s}$ and minimum of value $32 \mathrm{~Kb} / \mathrm{s}$ based on its QoS requirement, respectively, and let its allocated bandwidth is $45 \mathrm{~Kb} / \mathrm{s}$ which will be well with in $64 \mathrm{~Kb} / \mathrm{s}$ and $32 \mathrm{~Kb} / \mathrm{s}$ to guarantee the QoS requirement, then, $A_{i j}^{\min }=32 \leq A_{i j}^{\text {alloc }}=45 \leq A_{i j}^{\max }=64$.

Definition 3: The Applications are categorized into running, hand-off, and new applications, and each application may have multiple streams and request all or some of the $q$ resources based on its QoS requirement.

Running Application: An application which has been scheduled already and the network resources have been allocated to it in the cell is called a running application. Each of the running application's QoS requirement and allocated resources is shown as follows.

$$
\begin{aligned}
(Q A)_{R_{i}}=\{ & \left(R_{i 1}^{\max }, R_{i 1}^{\text {alloc }} R_{i 1}^{\min }\right) \\
& \left.\left(R_{i 2}^{\max }, R_{i 2}^{\text {alloc }}, R_{i 2}^{\min }\right), \ldots,\left(R_{i q}^{\max }, R_{i q}^{\text {alloc }}, R_{i q}^{\min }\right)\right\}
\end{aligned}
$$

for $i$ th running application.

$R_{i j}^{\min } \quad$ minimum value of $j$ th resource requirement of $i$ th running application;

$R_{i j}^{\max }$ maximum value of $j$ th resource requirement of $i$ th running application;

$R_{i j}^{\text {alloc }}$ allocated value of $j$ th resource to $i$ th running application.

Fig. 1 shows a single cell network model with mobile hosts (MHs) 4, 5, and 6 with running applications.

Hand-off Application: When a mobile user move from one cell to another cell then hand-off takes place. An application which is scheduled earlier and resources were allocated to it in the previous cell will request for connection establishment 
in the new cell, such an application is called as a hand-off application. Each of the hand-off application's QoS requirement and allocated resources is shown as follows.

$$
\begin{aligned}
(Q A)_{H_{i}}= & \left\{\left(H_{i 1}^{\max }, H_{i 1}^{\text {alloc }}, H_{i 1}^{\min }\right),\right. \\
& \left.\left(H_{i 2}^{\max }, H_{i 2}^{\text {alloc }}, H_{i 2}^{\min }\right), \ldots,\left(H_{i q}^{\max }, H_{i q}^{\text {alloc }}, H_{i q}^{\min }\right)\right\}
\end{aligned}
$$

for $i$ th hand-off application.

$H_{i j}^{\text {min }}$ minimum value of $j$ th resource requirement of $i$ th hand-off application;

$H_{i j}^{\max }$ maximum value of $j$ th resource requirement of $i$ th hand-off application;

$H_{i j}^{\text {alloc }}$ allocated value of $j$ th resource to $i$ th hand-off application before hand-off.

Mobile hosts 7 and 8 in Fig. 1 are hand-off from the neighboring cells with hand-off applications.

New Application: When a mobile user initiates an application within a cell and request for scheduling by providing the resource requirement, it is called a new application. Each of the new application's QoS requirement is shown as follows.

$$
\begin{aligned}
(Q A)_{N_{i}}= & \left\{\left(N_{i 1}^{\max }, N_{i 1}^{\text {alloc }}, N_{i 1}^{\min }\right)\right. \\
& \left.\left(N_{i 2}^{\max }, N_{i 2}^{\text {alloc }}, N_{i 2}^{\min }\right), \ldots,\left(N_{i q}^{\max }, N_{i q}^{\text {alloc }}, N_{i q}^{\min }\right)\right\}
\end{aligned}
$$

for $i$ th new application.

$N_{i j}^{\min }$ minimum value of $j$ th resource requirement of $i$ th new application;

$N_{i j}^{\max }$ maximum value of $j$ th resource requirement of $i$ th new application.

Allocated quantity of resources to new applications will be zero (i.e., $N_{i j}^{\text {alloc }}=0$ ). In Fig. 1 , MHs 1, 2, and 3 are requesting mobile host with new applications.

Definition 4: Available resource is a vector $\Theta=$ $\left[\Theta_{1}, \Theta_{2}, \ldots \Theta_{q}\right]$ indicating the amount of available quantities of the resources $1,2, \ldots, q$, respectively, after allocating the resources for the scheduled applications (we call the running applications and the applications which have been allocated resources for scheduling as scheduled applications) of the cell.

\section{B. Linear Programming Based Resource Reduction Principle}

In this subsection, we discuss the linear programming based resource reduction (LP-RR) principle used in the proposed AC scheme. The LP-RR optimally reduces the allocated quantity of each resources of already scheduled applications in a cell without effecting their QoS requirement and allocated resources $(Q A)$. This helps in obtaining large quantity of resources $(\Theta)$ for scheduling the remaining hand-off and new applications.

For the discussion of the principle, consider $r$ applications which have been scheduled in a mobile cell and the applications have been allocated some of the $q$ resources at a certain proportion. Let $X$ be the remaining applications to be scheduled. In order to schedule possible remaining applications $(X)$, which needs more resources, we need to determine the fair reduction of resources from the scheduled applications without violating their QoS requirement. The reduction of resources is carried out only if the total allocated quantity of resource is greater than the total minimum requirement of running applications by certain threshold value $(\gamma)$, i.e., $I F\left(\sum_{i=1}^{r} R_{i j}^{\text {alloc }}-\sum_{i=1}^{r} R_{i j}^{\text {min }}\right)>$ $\gamma_{j} \forall j \in[1, q]$, then the total allocated quantity of resource is reduced by certain quantity, which is fixed by the reduction parameter $\left(\Omega\right.$ ). (We have chosen the $\Omega_{j}$ value to vary between 0.9 to 0.7 , if the $\gamma_{j}$ value is $10 \%$ and above the maximum quantity of $j$ th resource.) Now, the problem reduces to the following in linear programming problem, which is given by

$$
\begin{aligned}
& \operatorname{maximize} \sum_{j=1}^{q} \sum_{i=1}^{r} c_{i j} R_{i j} \\
& \text { subject to } \sum_{i=1}^{r} R_{i j} \leq \Omega_{j}\left(P_{j}-\Theta_{j}\right), \quad \forall j \in[1, q] \\
& R_{i j}^{\min } \leq R_{i j} \leq R_{i j}^{\text {alloc }}, \quad \forall i \in[1, r], \forall j \in[1, q] \\
& R_{i j} \geq 0 . \quad \forall i \in[1, r], \forall j \in[1, q]
\end{aligned}
$$

where $R_{i j}$ 's are decision variables to be solved, which gives the newly allocated quantity of resources for running applications on solving the above LP problem. $c_{i j}$ 's are the weights chosen for $j$ th resource of $i$ th application based on the allocated quantity of resource with respect to maximum and minimum resource requirement. We define the weight function as follows:

$$
c_{i j}=\frac{R_{i j}^{\max }-R_{i j}^{\text {alloc }}}{R_{i j}^{\max }-R_{i j}^{\min }} \quad \text { if } R_{i j}^{\max }>R_{i j}^{\text {alloc }}
$$

and we choose $c_{i j}=0.05$ when $R_{i j}^{\text {alloc }}=R_{i j}^{\max }$.

$\Omega_{j}$ is a reduction parameter value for $j$ th resource to keep the resource reduction well within the requirement range. On solving above LP problem the increased available resources $\Theta_{j}$ are allocated to requesting hand-off and new applications using resource allocation procedure followed in Section IV-C.

We propose to solve the LP problem, given in (1), by ANNs. To formulate the LP problem in terms of neural networks, it is required to construct an appropriate computational energy function so that the lowest energy state will correspond to the desired solution of the LP problem [17]. Hence, the above LP problem is converted into a minimization equivalent in linear programming by adding slack and surplus variables, which are solved by using ANN. After reducing the LP problem given in (1) to the minimization LP problem, we get

$$
\begin{aligned}
\text { minimize }-\sum_{j=1}^{q} \sum_{i=1}^{r} c_{i j} R_{i j} & \\
\text { subject to } \sum_{i=1}^{r} R_{i j}+s_{j} & =\Omega_{j}\left(P_{j}-\Theta_{j}\right) \forall j \in[1, q] \\
R_{i j}+s l k_{i j} & =R_{i j}^{\text {alloc }} \quad \forall i \in[1, r], \forall j \in[1, q] \\
R_{i j}-s l k_{i j}^{\prime} & =R_{i j}^{\min } \quad \forall i \in[1, r], \forall j \in[1, q] \\
R_{i j} & \geq 0 \quad \forall i \in[1, r], \forall j \in[1, q]
\end{aligned}
$$

where $s_{j}, s l k_{i j}$ are slacks, and $s l k_{i j}^{\prime}$ is a surplus variable.

The LP equation given in (2) can be represented in more compact matrix form equation, as follows.

$$
\begin{gathered}
\operatorname{minimize} c^{T} x \\
\text { subject to } V x=b \\
0 \leq x \leq x_{\max }
\end{gathered}
$$


where the matrix $V \in \Re^{(2(r * q)+q) \times(3(r * q)+q)}$ is called the constraint matrix of the LP, the vector $c \in \Re^{3(r * q)+q}$ is the coefficient of objective function variables, $b=$ $\left[\Omega_{i}\left(P_{i}-\Theta_{i}\right)\left|i \in[1, q], R_{i j}^{\text {alloc }}, R_{i j}^{\min }\right| i \in[1, r], j \in[1, q]\right]$ is the right-hand side vector in the constraint equation, and $x=\left[R_{i j}, \Theta_{j}, s_{j}, s l k_{i j}, s l k_{i j}^{\prime} \mid i \in[1, r], j \in[1, q],\right]$ is the decision variable vector.

Above the LP problem given in (3) is solved for decision variables by using an ANN by designing its connection weight matrix $(W)$ and biasing thresholds $(\theta)$ [17]-[19], where $W=$ $-A V^{T} V$, and $\theta=A V^{T} b-B \exp (-C t) c$. The sigmoid activation function is used for neuron output. $A, B$, and $C$ are positive scalar parameters. The values chosen for the parameters during simulation are given in Section VI-A.

\section{LP-RR Based AC Algorithm}

To discuss the proposed LP-RR based AC policy, consider a mobile cell environment in which $r$ applications have been running and the resources are allocated to them at an instant of time.

Let $h$ hand-off and $n$ new applications request for scheduling at that instant of time. The admission of $h$ and $n$ applications may take place in any one of the following cases. The priority has been given to hand-off applications over the new applications.

Case 1-Accept All $h$ and $n$ Applications: This is possible if the available resources $(\Theta)$ are more than or equal to the sum of total allocated resources for $h$ hand-off applications and total minimum resource requirement of $n$ new applications.

$$
\text { i.e., } \Theta_{j} \geq\left(\sum_{i=1}^{h} H_{i j}^{\text {alloc }}+\sum_{i=1}^{n} N_{i j}^{\min }\right) \quad \forall j \in[1, q] \text {. }
$$

If (4) is satisfied, then allocate minimum resources to new applications and previously allocated resources to hand-off applications from the available resource $(\Theta)$. To allocate excess resources available (i. e., $\Theta_{j}-\left(\sum_{i=1}^{h} H_{i j}^{\text {alloc }}+\sum_{i=1}^{n} N_{i j}^{\text {min }}\right) \forall j \in$ $[1, q])$ to requesting applications well with in the requirement and to provide some fairness to the applications, we convert this problem to a linear programming problem, which is given as

$$
\operatorname{maximize} \sum_{j=1}^{q}\left(\sum_{i=1}^{h} \varphi_{i j} X_{i j}+\sum_{i=1}^{n} \psi_{i j} Y_{i j}\right)
$$

subject to $\sum_{i=1}^{h} X_{i j}+\sum_{i=1}^{n} Y_{i j}$

$$
\begin{aligned}
& \leq\left(\Theta_{j}-\left(\sum_{i=1}^{h} H_{i j}^{\text {alloc }}+\sum_{i=1}^{n} N_{i j}^{\min }\right)\right) \\
\forall j & \in[1, q], \\
0 \leq X_{i j} & \leq\left(H_{i j}^{\max }-H_{i j}^{\text {alloc }}\right) \forall i \in[1, h], \forall j \in[1, q] \\
0 \leq Y_{i j} & \leq\left(N_{i j}^{\max }-N_{i j}^{\min }\right) \forall i \in[1, n], \forall j \in[1, q] \\
X_{i j}, Y_{k j} & \geq 0 . \quad \forall i \in[1, h], \forall k \in[1, n], \forall j \in[1, q]
\end{aligned}
$$

where $0 \leq \varphi_{i j}<1$, and $0 \leq \psi_{i j}<1$ could be the relative importance or weight given to the $i$ th hand-off and new ap- plication for $j$ th resource, respectively. The relative importance may be given in terms of their resource requirement quantity, i.e., $\varphi_{i j}=\left(H_{i j}^{\max }-H_{i j}^{\text {alloc }}\right) / \Theta_{j}$ for hand-off applications and $\psi_{i j}=\left(N_{i j}^{\max }-N_{i j}^{\min }\right) / \Theta_{j}$ for new applications. $X_{i j}$ and $Y_{i j}$ are the decision variables to be solved for excess resources to get total allocated resources for hand-off and new applications, respectively.

The total allocated resources for hand-off and new applications is as follows.

$$
\begin{aligned}
R_{(r+i) j}^{\text {alloc }} & =H_{i j}^{\text {alloc }}+X_{i j} \forall i \in[1, h] \\
\forall j & \in[1, q] \text { for hand-off applications } \\
R_{(r+h+i)}^{\text {alloc }} & =N_{i j}^{\text {min }}+Y_{i j} \forall i \in[1, n] \\
\forall j & \in[1, q] \text { for new applications. }
\end{aligned}
$$

Here, we solve for $\Theta_{j}<\left(\sum_{i=1}^{h} H_{i j}^{\max }+\sum_{i=1}^{n} N_{i j}^{\max }\right)$; otherwise, the problem is trivial.

Case 2-Accept Possible Number of Hand-Off and New Applications: If the (4) is not satisfied, then Case 2 is considered. Here, the priority was given to hand-off applications over new applications. The resource allocation to the accepted applications are provided in the order of priorities: First, all hand-off applications are considered and later, the new applications using (6) and (7), respectively.

The resource allocation for hand-off applications is given by

$$
\begin{array}{r}
\text { While }\left(\left(\Theta_{j}-\sum_{k=1}^{i-1} H_{k j}^{\text {alloc }}\right)>H_{i j}^{\text {alloc }}, \forall j \in[1, q]\right) \\
R_{(r+i) j}^{\text {alloc }}=H_{i j}^{\text {alloc }} ; \quad \forall i \in[1, h]
\end{array}
$$

and for new applications

$$
\begin{aligned}
& \text { While }\left(\left(\Theta_{j}-\left(\sum_{k=1}^{h} H_{k j}^{\text {alloc }}+\sum_{l=1}^{i-1} N_{l j}^{\min }\right)\right)>N_{i j}^{\min }\right. \\
& \forall j \in[1, q]) \\
& R_{(r+h+i) j}^{\text {alloc }}=N_{i j}^{\text {min }} ; \forall i \in[1, n] .
\end{aligned}
$$

To accommodate possible number of remaining hand-off and new applications a linear programming based resource reduction with ANN model (described in Section IV-B) is used to reduce the allocated quantity of resources for running applications. The LP-RR updates the available resource $(\Theta)$ and are allocated to possible hand-off and new applications. Algorithm 1describes the LP-RR principle-based AC procedure.

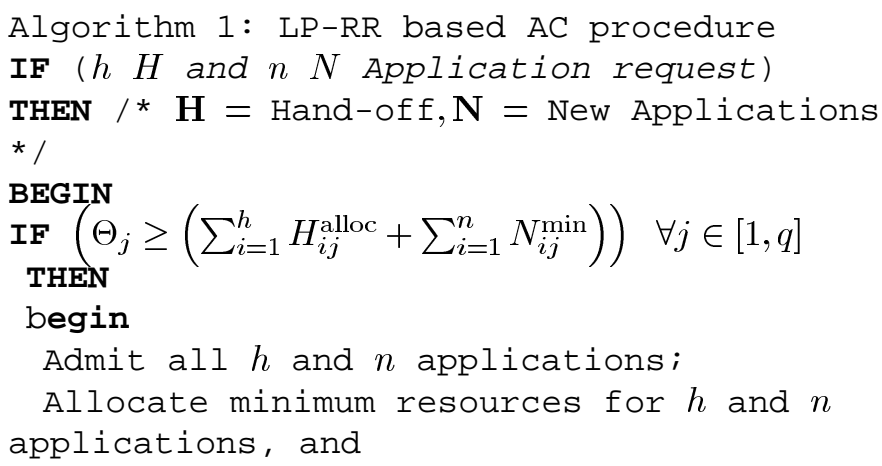




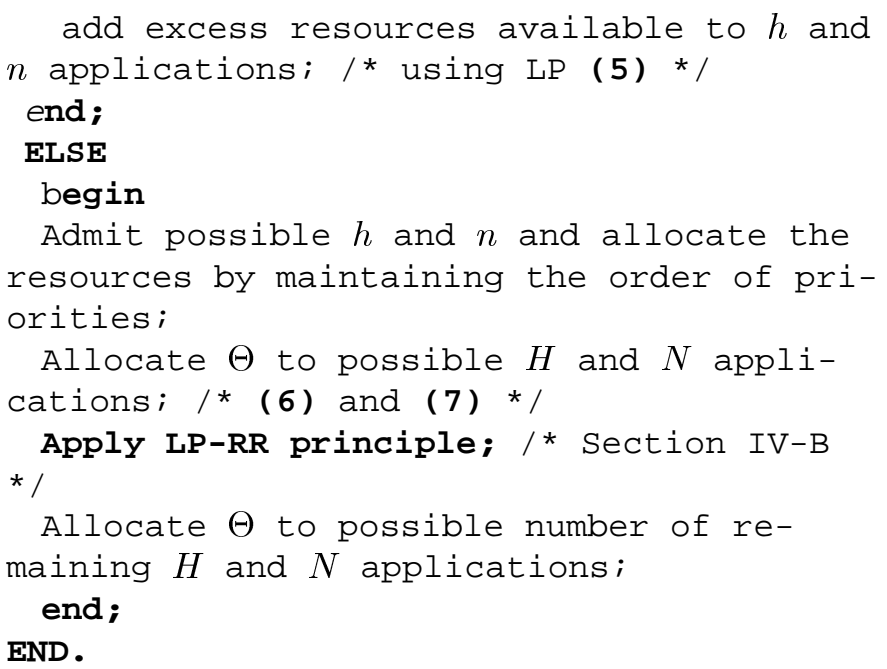

\section{Analytical Model}

In this section, we discuss the analytical model for the proposed LP-RR based AC scheme. We compute the performance of the AC policy for with and without resource reduction principle based on the following assumptions.

\section{A. Assumptions}

1) The applications requirements are independent of one another. These are aimed to get a set of resources.

2) The available system resources may or may not be sufficient to meet the minimal resource requirements of all the arrived applications. Hence, if the available quantity of resources are sufficient, then accept all the applications with required resource allocation to each one of them. Otherwise reject some of the applications based on the priority given to different applications. We have given the priority to hand-off applications over new applications.

3) The available quantity of resources $(\Theta)$ are uniformly distributed from zero to maximum available capacity. This assumption is considered to be reasonable because the availability of resources for different traffic conditions are unpredictable due to variable quantity of resource requirement of multimedia applications.

4) The minimum resource requirement of each applications are uniformly distributed from some minimum quantity to maximum quantity. This assumption is considered to be more practical because of the wide variety of resource requirement of user and multimedia applications.

\section{Notation:}

$m$

number of applications arrival at any instant of time (both hand-off and new applications);

$P_{j} \quad$ maximum capacity of $j$ th resource;

$\Theta_{j} \quad$ available quantity of $j$ th resource;



Fig. 2. Probability density function for the sum of applications minimum requirement for $m=2, m=4$, and $m=6$.

$M_{i j} \quad$ minimum resource requirement of $i$ th applica-

$\operatorname{Pr}(\cdot) \quad$ probability of $\cdot$;

$f(\cdot) \quad$ density function of $\cdot$;

$F(\cdot) \quad$ cumulative distribution function of $\cdot$;

$j \in[1, q], \quad i \in[1, m]$.

1) To Find the Probability of Sum of Minimum Resource Requirement of $m$ Arrived Applications: From assumption 4, the minimum resource requirement of each applications is considered to vary uniformly from $\min _{j}$ to $\max _{j}$, where $\min _{j}$ and $\max _{j}$ are the given parameter values for $j$ th resource of an application.

Here, $M_{i j}$ is a real and continuous random variable which is uniform $\left(\min _{j}, \max _{j}\right)$, then the probability of requirement is a density function of $M_{i j}$, which is given by

$$
f\left(M_{i j}\right)=\left\{\begin{array}{ll}
\frac{1}{\left(\max _{j}-\min _{j}\right)}, & \min _{j}<M_{i j}<\max _{j} \\
0, & \text { otherwise }
\end{array} \mid .\right.
$$

Here, $M_{i j}$ 's are $m$ independent and identically distributed random variables. The probability of sum of $m$ such random variables (i.e., the sum of $m$ applications minimum resource requirement for $j$ th resource) is given by:

For easy analysis, we normalize the requirement of each applications to lie between zero and one, i.e., $M_{i j}$ is uniform from 0 to 1 .

$$
\text { i.e., } M_{i j}=\frac{M_{i j}-\min _{j}}{\max _{j}-\min _{j}}, \forall i \in[1, m] \text {. }
$$

The compact equation for the density function of the sum of $m$ random variables, letting $M_{j}=\sum_{i=1}^{m} M_{i j}$, is shown in (9) at the bottom of the page, where $g\left(M_{j}\right)=\left\{\begin{array}{ll}0, & M_{j}<0 \\ M_{j}, & M_{j} \geq 0\end{array} \mid\right.$.

The graph shown in Fig. 2 gives the probability density function for $m=2,4$, and 6 applications.

2) To Find the Probability of Acceptance of Applications Given the Available Quantity of jth Resource: From the compact equation (9), the probability of acceptance of $k$ applications, given the available capacity $\Theta_{j}$ of $j$ th resource is obtained as follows.

$$
\begin{aligned}
\operatorname{Pr}\left(M_{j}\right) & =f_{m}\left(M_{j}\right) \\
& =\frac{m C_{0} \times\left[g\left(M_{j}\right)\right]^{m-1}-m C_{1} \times\left[g\left(M_{j}-1\right)\right]^{m-1}+\cdots+(-1)^{m} m C_{m} \times\left[g\left(M_{j}-m\right)\right]^{m-1}}{(m-1) !}
\end{aligned}
$$






Fig. 3. Probability of acceptance of applications for $\Theta_{j}=5, \Theta_{j}=7$, and $\Theta_{j}=9$.

Continuing with the normalization of resources requirement of each applications to lie between 0 and 1, the available quantity of resource $\left(\Theta_{j}\right)$ is normalized correspondingly, i.e., $\Theta_{j}=$ $\left(\Theta_{j}-k \cdot \min _{j}\right) /\left(\max _{j}-\min _{j}\right)$, since the minimum requirement of $k$ applications is $k \cdot \min _{j}$.

The probability of acceptance of $k$ applications is the same as the probability that the sum of the $k$ application's $j$ th resource requirement is less than or equal to $\Theta_{j}$, i.e.,

$$
\begin{aligned}
\operatorname{Pr}\left(M_{j} \leq \Theta_{j} \mid \Theta_{j}\right) & =F_{k}\left(\Theta_{j}\right) \\
& =\int_{-\infty}^{\Theta_{j}} f_{k}\left(M_{j}\right) d M_{j} \\
& =\int_{M_{j}=0}^{\Theta_{j}} f_{k}\left(M_{j}\right) d M_{j}
\end{aligned}
$$

since the available quantity of resources are real and continuous, and the minimum requirement of the applications are a positive real quantity, where $f_{k}\left(M_{j}\right)$ is the density function of the sum of the minimum requirement of $k$ applications given by (9), and $F_{k}\left(\Theta_{j}\right)$ is the cumulative distribution function (CDF) of $\Theta_{j}$.

Probability of acceptance of applications versus the number of applications for different values of available quantity of the $j$ th resource is as shown in Fig. 3, with the available quantity of 5,7 , and 9 units and with peak applications of arrival of 15.

3) To Find the Probability of Acceptance of Applications Given the Available Quantity of All the $q$ Resources: The probability of acceptance of $k$ applications, given the available quantity of all the $q$ resources $\Theta_{1}, \Theta_{2}, \ldots, \Theta_{q}$, which are independent, is obtained as follows.

The available quantity of resources $\Theta_{j}$ 's are normalized with respect to the normalization of resources requirement of each applications given in (8), i.e., $\Theta_{j}=\left(\Theta_{j}-k \cdot \min _{j}\right) /$ $\left(\max _{j}-\min _{j}\right)$, since the minimum requirement of $k$ applications is $k \cdot \min _{j}, \forall j \in[1, q]$.

The probability of acceptance of $k$ applications, given $\Theta_{1}, \Theta_{2}, \ldots, \Theta_{q}$, which are independent, is given by

$$
\begin{aligned}
\underset{k}{\operatorname{Pr}}= & \operatorname{Pr}_{k}\left(M_{1} \leq \Theta_{1} \wedge M_{2} \leq \Theta_{2} \ldots\right. \\
& \left.\wedge M_{q} \leq \Theta_{q} \mid\left(\Theta_{1}, \Theta_{2}, \ldots, \Theta_{q}\right)\right) \\
= & \prod_{j=1}^{q} \operatorname{Pr}\left(M_{j} \leq \Theta_{j}\right) .
\end{aligned}
$$

From (9) and (10), we obtain

$$
\begin{aligned}
\underset{k}{\operatorname{Pr}}=\int_{M_{1}=0}^{\Theta_{1}} f_{k}\left(M_{1}\right) d M_{1} \int_{M_{2}=0}^{\Theta_{2}} & f_{k}\left(M_{2}\right) d M_{2} \ldots \\
& \times \int_{M_{q}=0}^{\Theta_{q}} f_{k}\left(M_{q}\right) d M_{q}
\end{aligned}
$$

where $f_{k}\left(M_{j}\right)$ is the density function of sum of minimum requirement of $k$ applications for the $j$ th resource given by (9).

4) To Find the Probability of Acceptance of Hand-Off Applications Out of $m=h+n$ Arrived Applications: The probability of acceptance of $h$ hand-off applications, given the available quantity of all the $q$ resources $\Theta_{1}, \Theta_{2}, \ldots, \Theta_{q}$, is obtained as follows. Here, the hand-off applications are given highest priority over new applications. Taking the ratio of hand-off to total number of arrived applications as $z=h / m$, then $h=z \cdot m$.

By normalizing the resources requirement of each applications to lie between 0 and 1 , given by (8), the available quantity of resources $\Theta_{j}$ s are normalized correspondingly, i.e., $\Theta_{j}=\left(\Theta_{j}-z \cdot m \cdot \min _{j}\right) /\left(\max _{j}-\min _{j}\right)$, since the minimum requirement of $h$ applications is $h \cdot \min _{j}, \forall j \in[1, q]$.

From (10) and (11), we obtain that the probability of acceptance of $h$ hand-off applications given $\Theta_{1}, \Theta_{2}, \ldots, \Theta_{q}$ is

$$
\begin{aligned}
& \underset{h}{\operatorname{Pr}}= \underset{h}{\operatorname{Pr}}\left(M_{1} \leq \Theta_{1} \wedge M_{2} \leq \Theta_{2} \ldots\right. \\
&\left.\wedge M_{q} \leq \Theta_{q} \mid\left(\Theta_{1}, \Theta_{2}, \ldots, \Theta_{q}\right)\right) \\
&=\prod_{j=1}^{q} \operatorname{Pr}\left(M_{j} \leq \Theta_{j}\right) \\
&=\int_{M_{1}=0}^{\Theta_{1}} f_{h}\left(M_{1}\right) d M_{1} \int_{M_{2}=0}^{\Theta_{2}} f_{h}\left(M_{2}\right) d M_{2} \ldots \\
& \quad \times \int_{M_{q}=0}^{\Theta_{q}} f_{h}\left(M_{q}\right) d M_{q} .
\end{aligned}
$$

The graph in Fig. 4 shows the probability of acceptance of total hand-off applications versus $h / m$ or $(z)$ for different values of $m(m 1, m 2$, and $m 3$, where $m 1<m 2<m 3)$, and $q=2$.

5) To Find the Probability of Acceptance of New Applications Out of $m(=h+n)$ Arrived Applications: The probability of acceptance of $n$ new applications, given the available quantity of all the $q$ resources $\Theta_{1}, \Theta_{2}, \ldots, \Theta_{q}$, which are independent, is obtained as follows. Here, the new applications are given lowest priority as compared to hand-off applications. Hence, the acceptance of new applications means all the hand-off applications are accepted.

The available quantity of resources $\left(\Theta_{j}\right)$ are normalized correspondingly, i.e., $\Theta_{j}=\left(\Theta_{j}-(h+n) \cdot \min _{j}\right) /$ $\left(\max _{j}-\min _{j}\right)$, Since the minimum resource requirement of new applications means the sum of minimum requirement $h$ and $n$ applications and is $(h+n) \cdot \min _{j}, \forall j \in[1, q]$. 


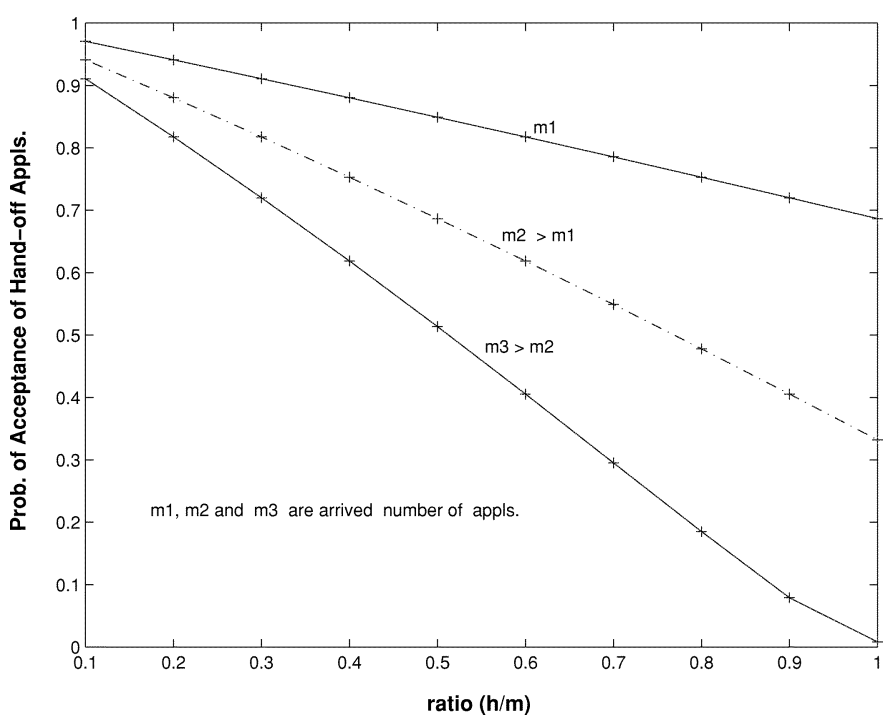

Fig. 4. Probability of acceptance of total hand-off applications for different values of $m$.

Then, the probability of acceptance of $n$ new applications is given by

$$
\begin{aligned}
& \underset{n}{\operatorname{Pr}}= \operatorname{Pr}\left(M_{1} \leq \Theta_{1} \wedge M_{2} \leq \Theta_{2} \ldots\right. \\
&\left.\wedge M_{q} \leq \Theta_{q} \mid\left(\Theta_{1}, \Theta_{2}, \ldots, \Theta_{q}\right)\right) \\
&=\prod_{j=1}^{q} \operatorname{Pr}\left(M_{j} \leq \Theta_{j}\right)
\end{aligned}
$$

where $M_{j}=\sum_{l=1}^{h} M_{l j}+\sum_{i=1}^{n} M_{i j}, \forall j \in[1, q]$.

From (10) and (11), we obtain

$$
\begin{aligned}
\underset{n}{\operatorname{Pr}}=\int_{r_{1}=0}^{\Theta_{1}} f_{n}\left(M_{1}\right) d M_{1} \int_{r_{2}=0}^{\Theta_{2}} & f_{n}\left(M_{2}\right) d M_{2} \ldots \ldots \\
& \times \int_{r_{q}=0}^{\Theta_{q}} f_{n}\left(M_{q}\right) d M_{q} .
\end{aligned}
$$

The graph in Fig. 5 shows the probability of acceptance of total new applications versus $n / m$ or $(1-z)$ for different values of $m(m 1<m 2<m 3)$ for $q=2$.

\section{B. Probability of Acceptance of Applications Without Resource Reduction (RR)}

Consider an instant of time at which $m=h+n$ applications request for resources for scheduling. To find the probability that $k$ applications are accepted out of $m(\geq k)$ arrived applications. The requesting $m$ applications are arranged in the order of priority mentioned above.

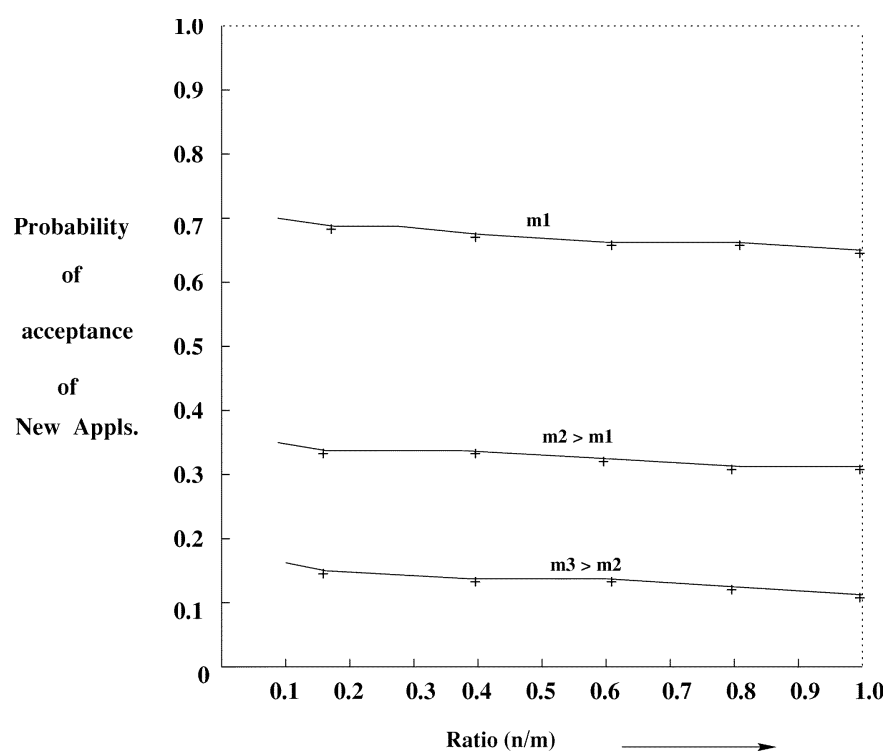

Fig. 5. Probability of acceptance of total new applications for different values of $m$.

From assumption 3, the available quantity of all the $q$ resources $\Theta_{1}, \Theta_{2}, \ldots, \Theta_{q}$ are uniformly distributed from zero to their respective maximum capacities and are independent. i.e., $\Theta_{j}$ is uniform from 0 to $P_{j}$ and is real and continuous.

Therefore, the probability of acceptance of $k$ out of $m$ applications requesting for certain quantities of the $q$ resources is given by the following. $\operatorname{Pr}^{q}(k)$ is the probability of available quantities of $q$ resources $\times$ the probability of sum of $k$ applications requirement over all the $q$ resources, where the probability of available quantity of $q$ resources is $\int_{0}^{P_{q}} \int_{0}^{P_{q-1}} \cdots \int_{0}^{P_{1}} \prod_{j=1}^{q}\left(f\left(\Theta_{j}\right) d\left(\Theta_{j}\right)\right)$, and the probability of the sum of $k$ applications minimum requirement over all the $q$ resources is $\int_{M_{q}=0}^{\Theta_{q}} \int_{M_{q-1}=0}^{\Theta_{q-1}} \ldots \int_{M_{1}=0}^{\Theta_{1}} \prod_{j=1}^{q}\left(f\left(M_{j}\right) d\left(M_{j}\right)\right)$. Hence, we have (14), shown at the bottom of the page.

The probability of acceptance of $k$ applications for $q=2$ is

$$
\begin{aligned}
\stackrel{2}{\operatorname{Pr}}(k)= & \int_{\Theta_{2}=0}^{P_{2}} \int_{\Theta_{1}=0}^{P_{1}} \operatorname{Pr}\left(\Theta_{1}\right) \operatorname{Pr}\left(\Theta_{2}\right) d\left(\Theta_{1}\right) d\left(\Theta_{2}\right) \\
& \int_{r_{2}=0}^{\Theta_{2}} \int_{r_{1}=0}^{\Theta_{1}} f\left(M_{1}\right) f\left(M_{2}\right) d\left(M_{1}\right) d\left(M_{2}\right) .
\end{aligned}
$$

For uniform distribution of $\Theta_{1}$ and $\Theta_{2},(15)$ reduces to

$$
\begin{aligned}
\stackrel{2}{\operatorname{Pr}}(k)= & \int_{\Theta_{2}=0}^{P_{2}} \int_{\Theta_{1}=0}^{P_{1}} \frac{1}{P_{1}} \cdot \frac{1}{P_{2}} d\left(\Theta_{1}\right) d\left(\Theta_{2}\right) \\
& \int_{M_{2}=0}^{\Theta_{2}} \int_{M_{1}=0}^{\Theta_{1}} f\left(M_{1}\right) f\left(M_{2}\right) d\left(M_{1}\right) d\left(M_{2}\right) .
\end{aligned}
$$

$$
\begin{aligned}
& \stackrel{q}{\operatorname{Pr}}(k)=\int_{0}^{P_{q}} \int_{0}^{P_{q-1}} \ldots \int_{0}^{P_{1}}\left(\prod_{j=1}^{q} f\left(\Theta_{j}\right)\right) d\left(\Theta_{1}\right) d\left(\Theta_{2}\right) \ldots d\left(\Theta_{q}\right) \\
& \int_{M_{q}=0}^{\Theta_{q}} \int_{M_{q-1}=0}^{\Theta_{q-1}} \ldots \int_{M_{1}=0}^{\Theta_{1}}\left(\prod_{j=1}^{q} f\left(M_{j}\right)\right) d\left(M_{1}\right) d\left(M_{2}\right) \ldots d\left(M_{q}\right)
\end{aligned}
$$




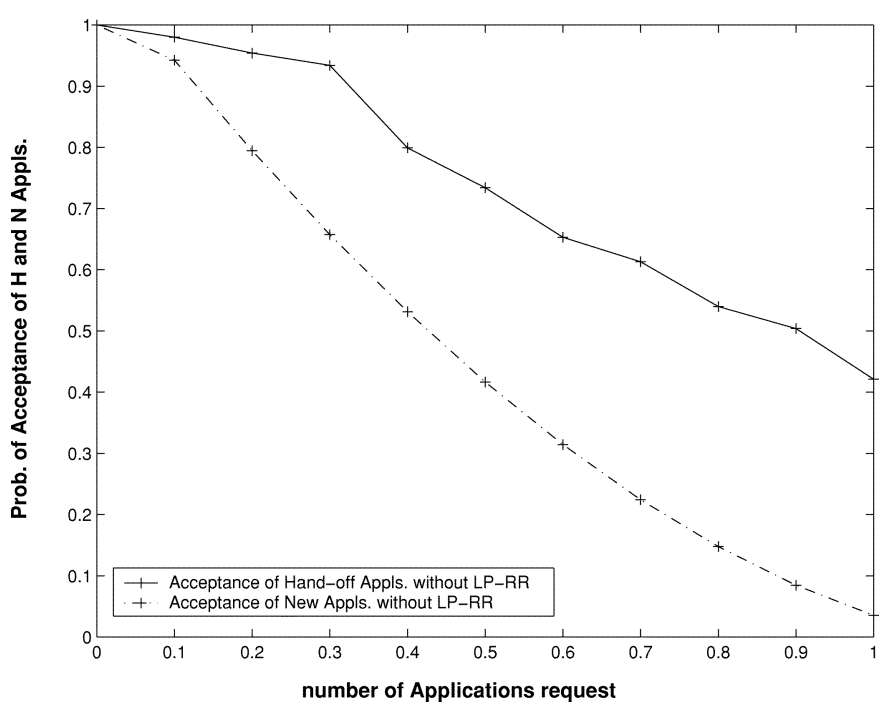

Fig. 6. Probability of acceptance of hand-off and new applications without resource reduction $(q=2)$.

The graph of probability of acceptance of applications versus number of applications with priority to hand-off applications over new applications, for $q=2$, is shown in Fig. 6 .

\section{Probability of Acceptance of Applications With Resource Reduction}

In this subsection, we discuss the probability of acceptance of applications with the resource reduction principle used in the proposed AC scheme.

For the discussion of the principle, consider $X$ applications which have been scheduled in a mobile cell and the applications have been allocated some of the $q$ resources at a certain proportion. Let $m$ be the remaining applications to be scheduled. In order to schedule possible remaining applications $(m)$, which needs more resources, it is fair to reduce a little quantity of allocated resources from the scheduled applications without violating their QoS requirement.

Consider an instant of time at which $m(=h+n)$ remaining applications are to be scheduled. To find the probability that $k$ applications are accepted out of $m(\geq k)$ remaining applications, in the order of priority mentioned above, let us consider that certain percentage $(P)$ of the allocated quantity of all the $q$ resources for running applications have been reduced using the LP-RR principle described in Section IV-B and, hence, that the

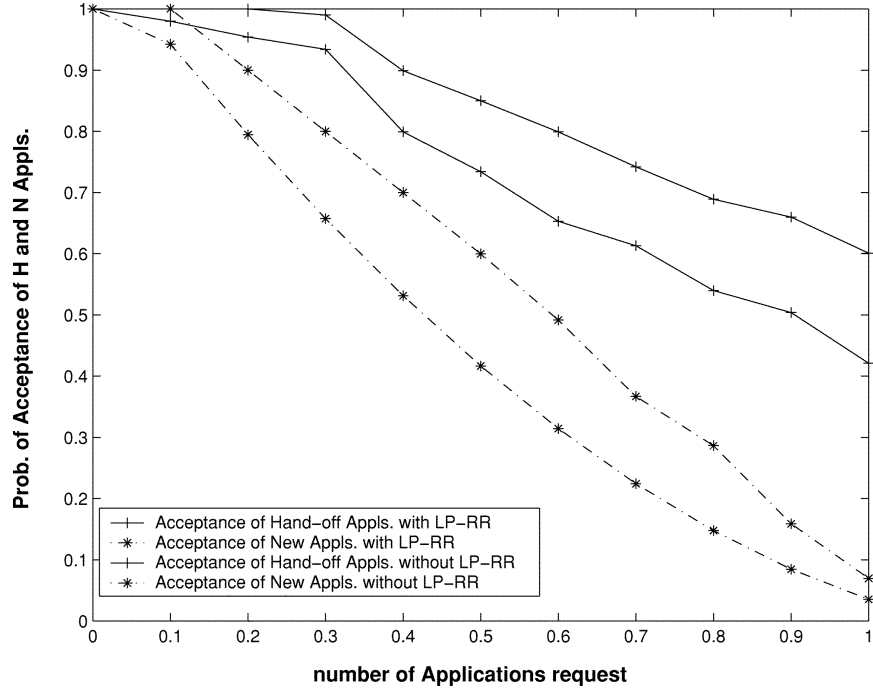

Fig. 7. Probability of acceptance of hand-off and new applications with and without resource reduction $(q=2)$.

available quantity of resources are increased correspondingly, as follows:

$$
\begin{aligned}
& \Theta_{j}^{\prime}=\Theta_{j}+P_{j}\left(P_{j}-\Theta_{j}\right) \text { for the } j \text { th resource } \\
& \Theta_{j}^{\prime}=\left(1-P_{j}\right) \Theta_{j}+\Omega_{j} P_{j}
\end{aligned}
$$

where $\Theta_{j}^{\prime}$ is the available quantity of the $j$ th resource after resource reduction.

When the available quantity $\Theta_{j}=0$, then $\Theta_{j}^{\prime}=P_{j} P_{j}$

Now, for the new values of available quantities of resources, the probability of acceptance of $k$ out of $m$ applications, by considering the assumptions made earlier, we get that $\operatorname{Pr}^{q}(k)$ is the probability of available quantity of resources after resource reduction $\times$ probability of sum of $k$ applications requirement, where we have the equation at the bottom of the page.

Hence, we have (17), shown at the bottom of the next page.

The graph of probability of acceptance of hand-off and new applications verses number of applications with and without resource reduction, for $q=2$, is shown in Fig. 7 .

\section{Simulation}

\section{A. Simulation Model Description and Parameter Values}

The simulation model is composed of seven cells, in which each cell keeps contact with its neighboring cells (see Fig. 8).

Probability of available quantity of resources after resource reduction

$$
=\int_{\Theta_{q}^{\prime}=P_{q} P_{q}}^{P_{q}} \int_{\Theta_{q-1}^{\prime}=P_{q-1} P_{q-1}}^{P_{q-1}} \cdots \int_{\Theta_{1}^{\prime}=P_{1} P_{1}}^{P_{1}} \prod_{j=1}^{q}\left(f\left(\Theta_{j}^{\prime}\right) d\left(\Theta_{j}^{\prime}\right)\right)
$$

Probability of sum of $k$ applications requirement over all $q$ resources

$$
=\int_{M_{q}=0}^{\Theta_{q}^{\prime}} \int_{M_{q-1}=0}^{\Theta_{q-1}^{\prime}} \cdots \int_{M_{1}=0}^{\Theta_{1}^{\prime}} \prod_{j=1}^{q}\left(f\left(M_{j}\right) d\left(M_{j}\right)\right)
$$




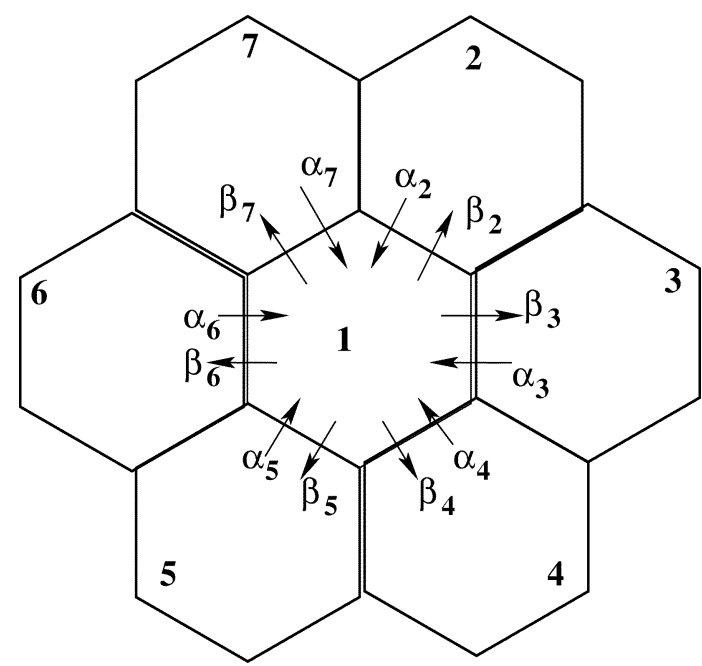

Fig. 8. Seven cell network simulation model.

Each cell contains a base station, which is responsible for scheduling new applications and hand-off applications. Also, the base station controls the resource redistribution to running applications using the LP-RR principle when the request for scheduling from new and hand-off application arrives.

The simulation model is designed to consider the assumptions that were considered in the analytical model and to emulate the realistic mobile cell environment that consists of data and real-time application traffic. The traffic generator is simulated by considering random arrival of applications with their resource requirement, uniformly distributed in certain range to represent realistic multimedia applications.

In the simulation, as mentioned in the proposed scheme, we consider two types of application traffic: a new application traffic, which is initiated by a mobile user within a cell and a hand-off application traffic, which is handed off from the neighboring cells, as shown in Fig. 8. The total load in a cell are the running applications at a given instant of time. At that instant of time, the following events will initiate our AC procedure with LP-RR principle:

- Request from hand-off applications due to mobile hosts moving out from the neighboring cells toward cell 1 . In the simulation model, we consider that there are six neighboring cells, each having a load of $l_{i},(i=2,3, \ldots, 7)$ applications out of which $\alpha_{i}(i=2,3, \ldots, 7)$ times their total running applications are hand-off toward the cell 1 (see Fig. 8); then, the total number of hand-off applications in cell $1=\alpha_{2} l_{2}+\alpha_{3} l_{3}+, \ldots,+\alpha_{7} l_{7}$. Here, $\alpha$ values depend on the mobile host's mobility behavior, which is beyond the scope of this work. The same situation replicates for other cells in the system, but in the simulation, we have taken different values for $\alpha$ to show the performance of the system for high and low mobility environment.

- Due to mobile host moving out of the cell 1 , the load within the cell reduces and correspondingly the resources availability increases. In Fig. 8 , it is shown that $\beta_{i}$, $(i=2,3, \ldots, 7)$, times the total running applications are moving out of the cell 1 . Here again, $\beta$ values depend on the mobile hosts mobility behavior.

- Request from new applications initiated by the mobile hosts within a cell 1 .

- Due to termination of the applications, the load within a cell reduces by increasing the available quantity of resources.

Hence, at any instant of time, one or more of the above events may occur. In the simulation model we have considered the events with uniform distribution for arrival of hand-off and new applications, terminating, and outgoing applications. During termination and outgoing events, the resources are released and used for allocation to requesting hand-off and new applications. Whenever the request from hand-off and new applications arrives, our model admit the possible applications with priority to hand-off applications and if any remaining applications then the LP-RR principle is applied to admit more applications.

In the simulation, the new and hand-off applications are generated by considering some random values to $\alpha$ 's at an instant of applications arrival. We consider the total number of applications, both hand-off $\sum_{i=2}^{7} \alpha_{i} l_{i}$ and new applications, arrival to be distributed uniformly from single application up to a certain maximum value.

For simulation, two of the resources $(q=2)$ are considered, for example, bandwidth (BW) and buffer having a maximum capacity of $100 \mathrm{Mb} / \mathrm{s}$ and $500 \mathrm{Mbytes}$, respectively, i.e., $P_{1}=$ $100 \mathrm{Mb} / \mathrm{s}$ and $P_{2}=500$ Mbytes.

In order to represent various multimedia application traffic, we consider the bandwidth and buffer requirement of each applications to vary uniformly in the maximum and minimum requirement range.

In the simulation, based on the maximum capacity of resources (BW and buffer) and the number of applications arrival at any instant of time, the maximum bandwidth and buffer requirement of applications is considered uniformly distributed in the range 0.5 to $5 \mathrm{Mb} / \mathrm{s}$ and 2 to 20 Mbytes, respectively. The minimum quantity of resource requirement is considered from 90 to $0 \%$ of the maximum resource requirement to realize applications QoS requirement and tolerance, as a realistic traffic (shown in Table I).

$$
\begin{array}{r}
\stackrel{q}{\operatorname{Pr}(k)=\int_{\Theta_{q}^{\prime}=P_{q} P_{q}}^{P_{q}}} \int_{\Theta_{q-1}^{\prime}=P_{q-1} P_{q-1}}^{P_{q-1}} \ldots \int_{\Theta_{1}^{\prime}=P_{1} P_{1}}^{P_{1}}\left(\prod_{j=1}^{q} f\left(\Theta_{j}^{\prime}\right) d\left(\Theta_{i}^{\prime}\right)\right) \\
\int_{M_{q}=0}^{\Theta_{q}^{\prime}} \int_{M_{q-1}=0}^{\Theta_{q-1}^{\prime}} \ldots \int_{M_{1}=0}^{\Theta_{1}^{\prime}}\left(\prod_{j=1}^{q} f\left(M_{j}\right) d\left(M_{j}\right)\right)
\end{array}
$$


TABLE I

PARAMETER VALUES OF SimUlation For AC

\begin{tabular}{c||c}
\hline Description & Value \\
\hline \hline Maximum available capacity of bandwidth & $100 \mathrm{Mbps}$ \\
\hline Maximum available capacity of buffer & $500 \mathrm{Mbytes}$ \\
\hline Range of Reduction parameter $(\Omega)$ & 0.7 to 0.9 \\
\hline Maximum requirement of bandwidth by an application & 0.5 to $5 \mathrm{Mbps}$ \\
\hline Maximum requirement of buffer by an application & 2 to $20 \mathrm{Mbytes}$ \\
\hline No. of (hand-off and/or new) appls. at every arrival event & 1 to 50 \\
\hline
\end{tabular}

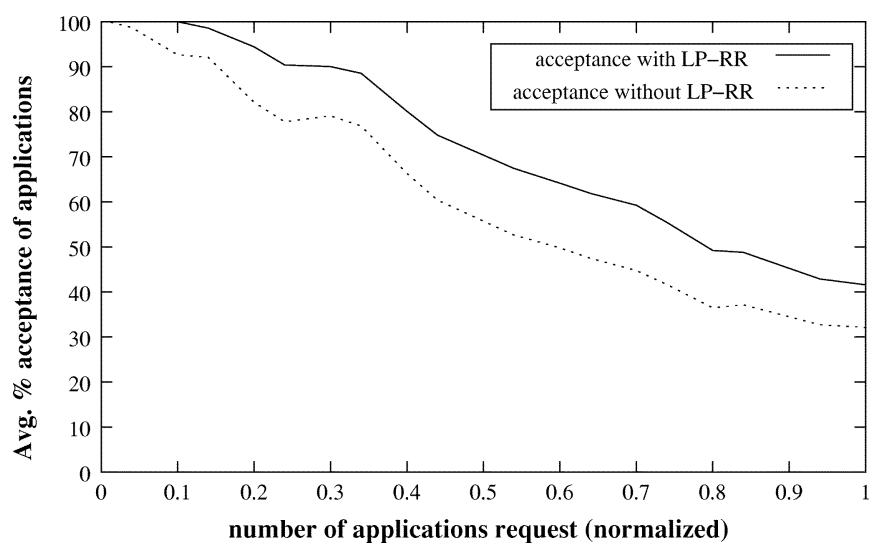

Fig. 9. Average percentage acceptance of applications.

The reduction parameter $\Omega$ value in constraint equation (1) of the LP-RR principle is carefully chosen to keep the resource reduction well within the requirement range, i.e., a graceful degradation of applications QoS requirement and allocated resources (QA). We choose in the simulation the $\Omega$ value to vary from 0.7 to 0.9 depending on the threshold value $\gamma$. Table I summarizes the parameter values used for simulation.

In the simulation model an LP-RR principle is applied to accommodate remaining applications during $\mathrm{AC}$ decision. The LP-RR principle uses the ANN model to solve LP problem.

The parameter values for the neural network are chosen, depending on the maximum value of the decision variables, to best fit the model. The parameter values are $B=A=300$ and $C=30$. The performance of ANN for resource reduction is investigated for fairness during the reduction of allocated resources for running applications for different values of resources requirement.

The simulation is carried out for 20000 arrival events with random number of application arrivals (peak arrival value $=$ 50) with random resource requirement (with in the specified requirement range) of each requesting applications for to realize the realistic network traffic. The performance measures obtained through the simulation are the average percentage acceptance of hand-off applications and new applications traffic, as well as the percentage utilization of bandwidth and buffer. The simulation results for the performance measures are plotted as a function of number of applications arrival (normalized with respect to peak arrivals).

In order to evaluate the performance of the proposed scheme, the simulation is also carried out for without $L P-R R$ principle by maintaining priority and equal priority among hand-off and new applications.

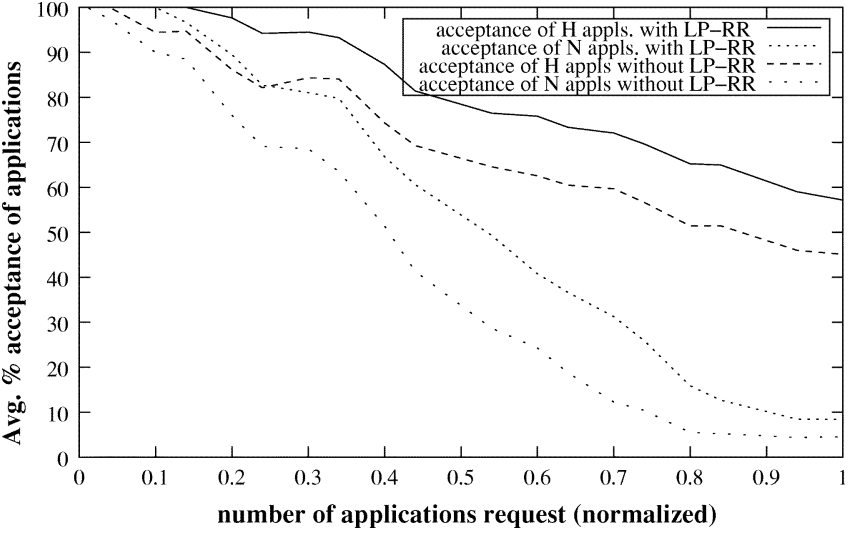

Fig. 10. Percentage acceptance of hand-off and new applications.

In addition, the performance of LP-RR principle is investigated for fairness during the reduction of allocated resources for running applications with different amount of allocated quantity of resources.

\section{B. Simulation Results}

Fig. 9 shows the graph for an average percentage acceptance of applications requesting for scheduling by providing the resource requirement for the simulation results. The average percentage acceptance is plotted with respect to the number of applications request. The results are plotted for with and without LP-RR principle and obtained a higher percentage of acceptance of requesting applications (about 10 to 15\%) for the scheme with LP-RR principle with an increased number of applications arrival. The percentage of acceptance has decreased up to $32 \%$ at peak arrivals of requesting applications without the LP-RR principle, whereas it is $42 \%$ with the LP-RR principle.

Fig. 10 shows the percentage acceptance of hand-off and new applications for the simulation results with priority to hand-off applications over new applications. It is clear from the graph that average acceptance for hand-off applications is approximately $45 \%$ more than that of new applications at peak arrivals. The simulation is carried out with and without LP-RR principle, for which the average percentage acceptance has decreased 10 to $15 \%$ more for AC scheme without LP-RR principle as compared to scheme with LP-RR principle.

The graph of percentage utilization of bandwidth and buffer is shown in Fig. 11, where the utilization has been increased up to $90 \%$ at $50 \%$ of the peak arrivals and maintained an almost constant utilization up to peak arrivals, because the AC scheme uses an LP-RR principle for resource allocation to the running and requesting hand-off and new applications. The resource utilization is increased by 7 to $12 \%$ for requesting applications with LP-RR principle than without the LP-RR principle from 50 to $100 \%$ of the peak applications arrival.

The pie charts shown in Fig. 12(a) and (b) gives the percentage of admitted and rejected hand-off and new applications out of cumulative sum of arrived hand-off and new applications for the total simulation run (i.e., for 20000 arrival events). The percentage of admitted hand-off is $52 \%$, and new applications is $8 \%$ with the LP-RR principle, where as it is 42 and 5\% for hand-off and new applications respectively, without the LP-RR principle. 


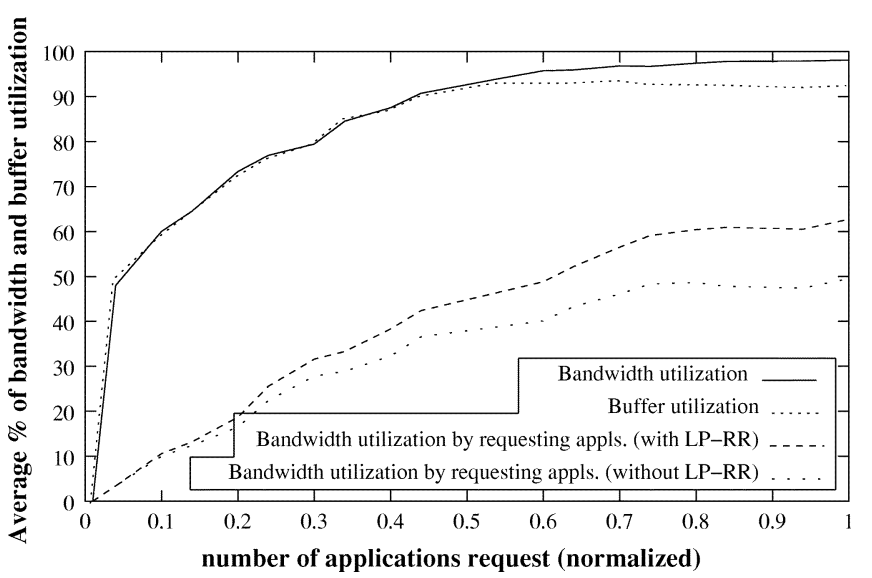

Fig. 11. Total percentage of bandwidth and buffer utilization and bandwidth utilization by requesting applications.

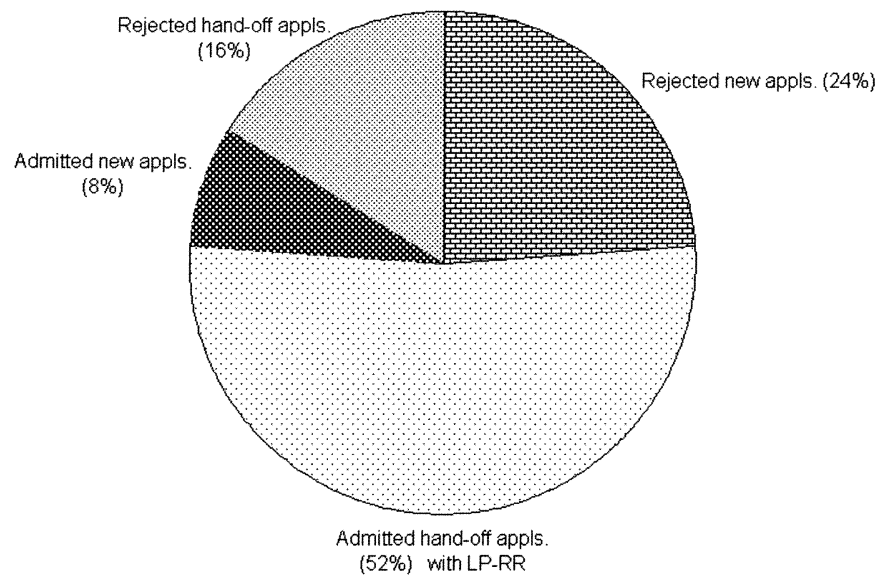

(a)



(b)

Fig. 12. Total percentage of admitted and rejected hand-off and new applications. (a) With LP-RR principle. (b) Without LP-RR principle.

1) Fairness in Resource Reduction: Fig. 13 shows the graph of percentage reduction of allocated resources for running applications with respect to their maximum resource requirement and their requirement range. The graphs are plotted for the results taken from the ANN model with different resource requirement and requirement range for running applications. The curve shows that the resource reduction is proportional to the applications requirement and requirement range. The

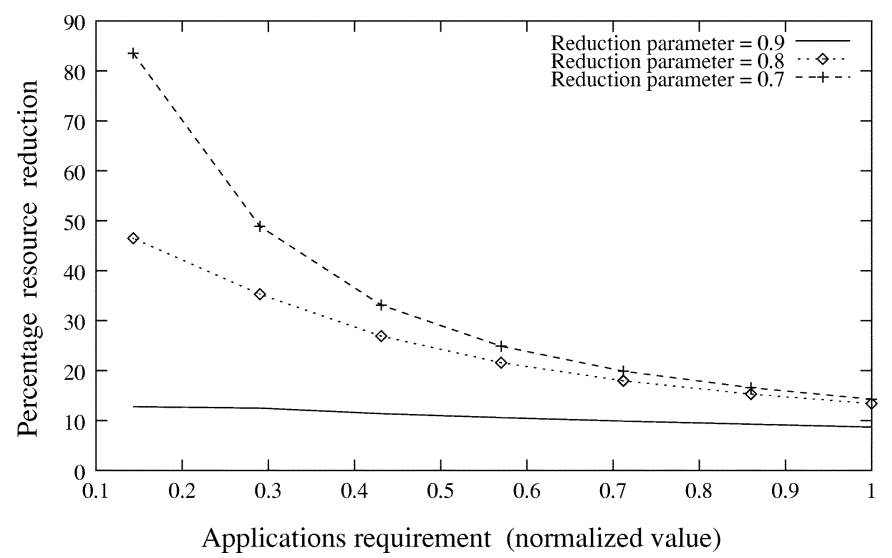

(a)

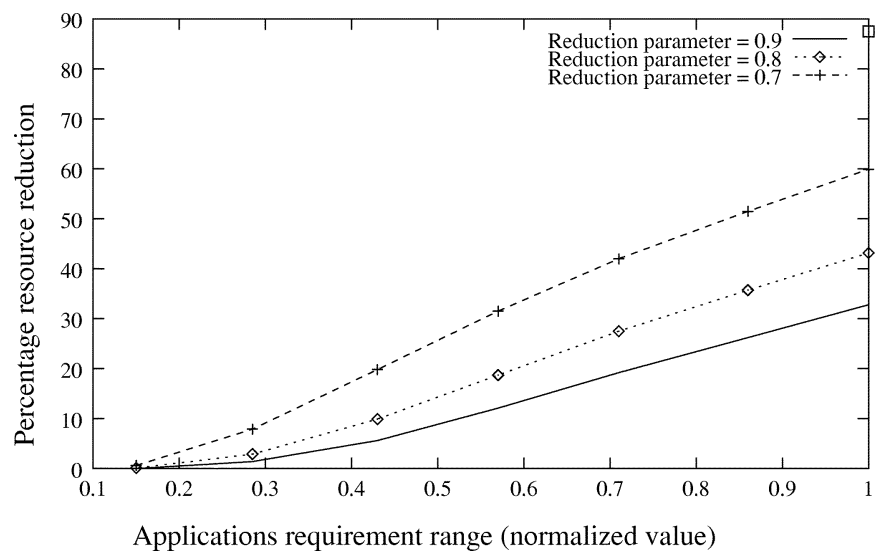

(b)

Fig. 13. Percentage reduction of resources with respect to (a) maximum requirement with fixed requirement range and (b) requirement range with fixed maximum requirement of applications.

graph in Fig. 13(a) shows that as the resource requirement of applications increases, with constant requirement range (i.e., maximum-minimum), the percentage of resource reduction decreases. The graph in Fig. 13(b) shows that as the requirement range increases, the percentage of resource reduction increases. From the above discussion, it is clear that some fairness is maintained between the applications of different resource requirement and range of requirement.

From the above simulation results, it is clear that the proposed AC scheme with LP-RR principle has shown an improved performance in terms of average resource utilization by requesting applications and average percentage of acceptance to hand-off and new applications as compared to the AC scheme without LP-RR principle. Since the priority was given to hand-off applications, it resulted in lower percentage of acceptance of new applications.

Also, a graph is plotted for acceptance of applications for both analytical and simulation results by considering average percentage of acceptance of applications in case of simulation model (normalized value) and probability of acceptance in case of analytical model, with respect to the number of applications request (see Fig. 14). From the graph, it clear that both analytical and simulation results are almost coinciding for acceptance of hand-off and new applications with and without LP-RR principle. 


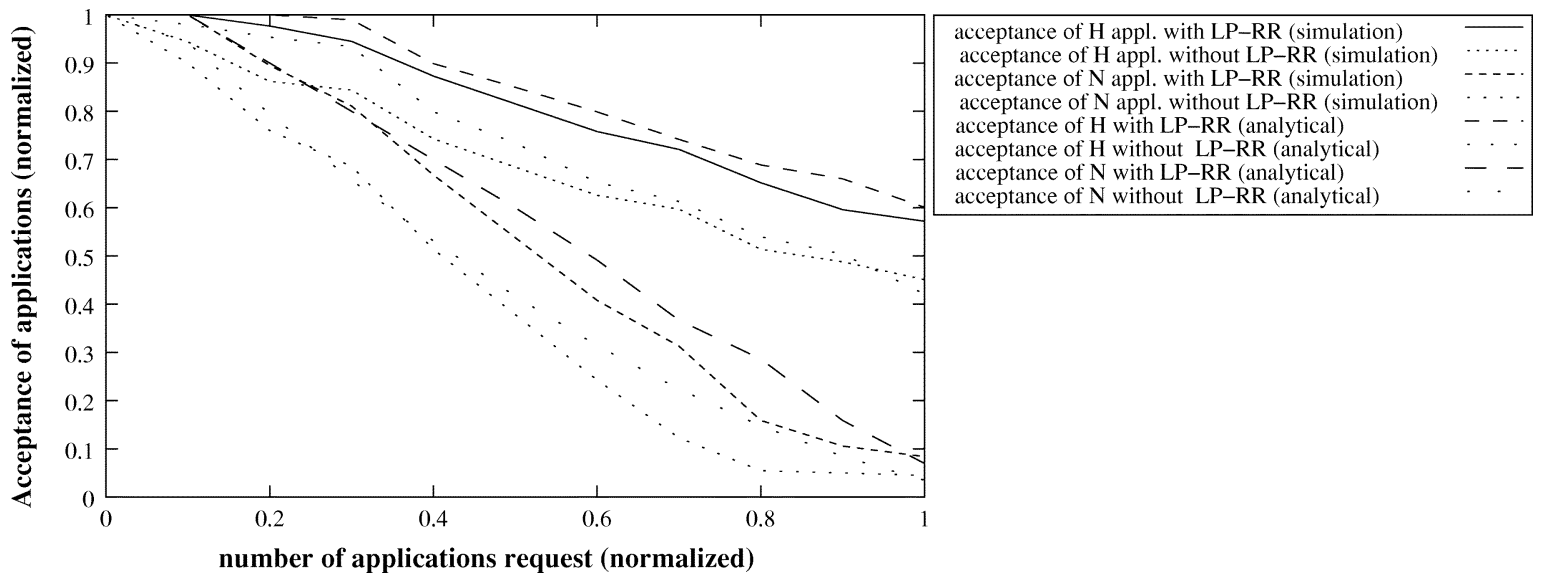

Fig. 14. Comparison of analytical and simulation results for acceptance of hand-off and new applications with and without LP-RR principle.

\section{CONCLUSION}

In this paper, an LP-RR principle-based AC for a mobile network has been proposed. The scheme provides priority to hand-off applications over new applications request for scheduling by providing the required amount of resources. The AC decision is based on QoS requirement (i.e., resource requirement ) of requesting applications. The LP-RR principle-based AC with resource allocation to running and requesting applications has improved the percentage acceptance of requesting applications. The LP-RR principle has shown a good performance in computational model. The ANN is capable of generating optimal solutions to linear programming problems. The easy implementation of neural networks in an analog circuits and potentially powerful computational model for solving real-time and largescale linear programming problems will help in practical system.

The results of our analytical model have been verified with simulation results. The simulation results agreed well with those of the analytical results for acceptance of hand-off and new applications with and without LP-RR principle.

It is shown through simulation results that the proposed scheme has increased the percentage of acceptance to requesting applications as compared to the $\mathrm{AC}$ scheme without LP-RR principle. Also, from the results it is verified that the LP-RR principle has maintained some fairness during the reduction of allocated resources for running applications.

\section{REFERENCES}

[1] W. C. Y. Lee, "Smaller cells for greater performance," IEEE Commun. Mag., vol. 29, pp. 19-23, Nov. 1991.

[2] W. B. Yang and E. Geraniotis, "Admission policies for integrated voice and data traffic in CDMA packet radio networks," IEEE J. Select. Areas Commun., vol. 12, pp. 654-664, May 1994.

[3] M. Naghshineh and A. S. Acampora, "QoS provisioning in micro-cellular networks supporting multimedia traffic," in Proc. IEEE INFOCOM, Boston, MA, Apr. 1995.

[4] M. Naghshineh and M. Schwartz, "Distributed call admission control in mobile/wireless networks," IEEE J. Select. Areas Commun., vol. 14, pp. 711-717, May 1996.

[5] J. Tajima and K. Imamura, "A strategy for flexible channel assignment in mobile communication systems," IEEE Trans. Veh. Technol., vol. 37, pp. 92-103, May 1988.

[6] D. Hong and S. S. Rappaport, "Traffic model and performance analysis of cellular radio telephone systems with prioritized and nonprioritized hand-off procedures," IEEE Trans. Veh. Technol., vol. VT-35, pp. 77-92, Aug. 1986.
[7] S. H. Oh and D. W. Tcha, "Prioritized channel assignment in a cellular radio network," IEEE Trans. Commun., vol. 40, pp. 1259-1269, July 1992.

[8] S. Tekinay and B. Jabbari, "A measurement based prioritization scheme for handover in mobile cellular networks," IEEE J. Select. Areas Commun., vol. 10, pp. 1343-1350, Oct. 1992.

[9] I.-R. Chen and T. H. Hsi, "Performance analysis of admission control algorithms based on reward optimization for real-time multimedia servers," Performance Evaluation, vol. 33, no. 2, pp. 89-112, July 1998.

[10] D. Levine, I. Akyildiz, and M. Naghshineh, "A resource estimation and call admission algorithm for wireless multimedia networks using the shadow cluster concept," IEEE/ACM Trans. Networking, vol. 5, pp. 1-12, Feb. 1997.

[11] D. Ayyagari and A. Ephremides, "Admission control with priorities: Approaches for multi-rate wireless systems," Mobile Networks Applicat., vol. 4, pp. 209-218, 1999.

[12] G. Ramamurthy and Q. Ren, "Multi-class connection admission control policy for high speed ATM switches," in Proc. IEEE INFOCOM, vol. 3, Kobe, Japan, Apr. 1997, pp. 963-972.

[13] A. K. Talukdar, B. R. Badrinath, and A. Acharya, "On accommodating mobile hosts in an integrated services packet networks," in Proc. INFOCOM, vol. 3, Kobe, Japan, Apr. 1997, pp. 1046-1053.

[14] C. Lee, "On quality of service management," Ph.D. dissertation, Carnegie Mellon Univ., Pittsburgh, PA, 1999.

[15] J. M. Peha and A. Sutivong, "Admission control algorithm for cellular systems," Wireless Networks, vol. 7, pp. 117-125, 2001.

[16] D. Zhao, X. Shen, and J. W. Mark, "QoS performance bounds and efficient connection admission control for heterogeneous services in wireless cellular networks," Wireless Networks, vol. 8, pp. 85-95, 2002.

[17] J. Wang and V. Chankong, "Recurrent neural networks for linear programming: Analysis and design principles," Comput. Oper. Res., vol. 19, pp. 297-311, 1992.

[18] J. J. Hopfield and D. W. Tank, "Neural computation of decisions in optimization problems," Biolog. Cybern., vol. 52, pp. 141-152, 1985.

[19] J. Wang, "Analysis and design of a recurrent neural network for linear programming," IEEE Trans. Circuits Syst. I, vol. 40, pp. 613-618, Sept. 1993.

B. P. Vijay Kumar (S'00) received the B.E. degree in electronics and communication from Mysore University, Mysore, India, in 1988 and the M.Tech. degree in computer science and technology with honors from the Indian Institute of Technology, Roorkee, India, in 1992.

$\mathrm{He}$ is an Assistant Professor of Computer Science and Engineering with the Jawaharlal Nehru National College of Engineering, Vishweshwarayya Technological University, Shimoga, Karnataka, India. Currently, he is pursuing the Ph.D. degree with the Electrical Communication Engineering Department, Indian Institute of Science, Bangalore, India. His area of research is neural network applications in mobile networks. His interested areas of research are mobile networks, protocol engineering, and computational intelligence technique applications in mobile networks.

Mr. Kumar is a student member of the IEEE Computer Society and a Life Member of the India Society for Technical Education (LMISTE). 
Pallapa Venkataram (M'91-SM'99) received the M.Sc. degree in mathematics from Sri Venkateswara University, Tirupathi, India, in 1973 and the Ph.D. degree in information sciences from the University of Sheffield, Sheffield, U.K., in 1986. He also received the Distinguished Visitor Diploma from the Orrego University, Trujillo, Peru.

He is currently a Professor of electrical communication engineering with the Indian Institute of Science, Bangalore, India, where he is involved in wireless access research and protocol engineering. He was with Hindustan Aeronautical Limited, Bangalore, as a Deputy Manager (Computers) from 1980 to 1982, where he worked mainly on systems design for financial and material management systems. He was a Visiting Scientist at the University of Maryland, Baltimore, and at the University of Montreal, Montreal, QC, Canada, in 1992. In 1995, he was a Visiting Scientist at the SK University, Anantapur, India. In 1998, he spent six months with Northern Telecom (NORTEL), Ottawa, ON, Canada, on sabbatical. He serves on several advisory committees and frequently consults to government of India and industry. His current research interests are in the areas of communication protocols, wireless networks, and computational intelligence applications in communication networks and multimedia systems. He has published around 150 papers, and he has edited a book entitled Wireless Communications for the Next Millennium. He has written a chapter in the book Computational Intelligence in Telecommunications Networks and co-authored a chapter in the book Mobile Multimedia Communications.

Dr. Venkataram received a Communication Devices India Ltd., (CDIL) Award for a paper published in the IETE Journal. Dr. Venkataram has served in various capacities in many IEEE and ICCC conferences and workshops. He is a Fellow of Institution of Electrical Engineers (IEE), U.K., a Fellow of the Institution of Electronics and Telecommunication Engineers (IETE), India, and is a senior member of the IEEE Computer Society. 